\title{
Recent H-mode density limit studies at JET
}

\author{
K. Borrass ${ }^{1}$, A. Loarte $^{2}$, C.F. Maggi ${ }^{1}$, V. Mertens ${ }^{1}$, P. Monier ${ }^{3}$, \\ R. Monk ${ }^{1}$, J. Ongena ${ }^{4}$, J. Rapp ${ }^{5}$, G. Saibene ${ }^{2}$, R. Sartori ${ }^{2}$, \\ J. Schweinzer ${ }^{1}$, J. Stober ${ }^{1}$, W. Suttrop ${ }^{1}$ and EFDA-JET \\ Workprogramme collaborators ${ }^{\mathrm{a}}$ \\ ${ }^{1}$ Max-Plank-Institut für Plasmaphysik, Euratom Association, Garching, Germany \\ ${ }^{2}$ EFDA Close Support Unit Garching, Garching, Germany \\ ${ }^{3}$ Association EURATOM-CEA, CEA Cadarache, St Paul-Lez-Durance, France \\ ${ }^{4}$ Laboratoire de Physique des Plasmas, Ecole Royale Militaire, Euratom Association, \\ Brussels, Belgium \\ ${ }^{5}$ EFDA Close Support Unit Culham, Culham, UK
}

Received 28 October 2003, accepted for publication 6 May 2004

Published 27 May 2004

Online at stacks.iop.org/NF/44/752

DOI: 10.1088/0029-5515/44/7/007

\begin{abstract}
Extensive studies of the H-mode density limit (DL) in JET gas-puffed discharges have been performed in the past four years targeting at an improved database for extrapolation to ITER. This paper reviews the arguments for the particular DL definition (pedestal density at the H-L boundary), the logic underlying the choice of parameters under focus (toroidal field, major radius, triangularity, safety factor) as well as some improvement in the interpretation of typical JET density ramp-up signatures that led to a critical review of the existing data. An empirical scaling is derived and compared with existing empirical and model based scalings. ASDEX Upgrade data are included in this analysis to provide information on the size dependence. The main results are: earlier findings on the $B_{t}, R$ and $q_{95}$-dependences are confirmed. The triangularity dependence, if any, is weak. The SOL-based BLS (Borrass, Lingertat, Schneider) scaling and the empirical scaling are virtually indistinguishable. The Greenwald scaling provides values in the right absolute range, but the overall fit is comparatively poor. The proposed scaling predicts ITER critical densities considerably below the reference value. Fuelling methods other than gas-puffing are outside the scope of this paper, but pellet fuelling, envisaged for ITER as an option to alleviate the situation, is discussed.
\end{abstract}

PACS numbers: 52.55.Fa, 28.52.Cx

(Some figures in this article are in colour only in the electronic version)

\section{Introduction}

Traditionally the concept of a density limit (DL) is applied to the highest density achieved in a discharge which is actively fed with particles (density ramp-up). DLs can be attributed to specific tokamak operation regimes (L-mode DL, H-mode DL). Furthermore, the highest density may be achieved at the high-density boundary of the operation regime, so that the DL coincides with a regime transition (H-L transition H-mode DL, disruptive L-mode DL), or it may be manifested as resilience to further density increase (soft DL). Finally, the limit can be formulated as a limit for

a See appendix of the paper by J. Pamela 2000 Overview of recent JET results Proc. of the IAEA Conf. on Fusion Energy (Sorrento, 2000). certain moments of the density profile (line average, volume average) or for values taken at certain radial positions (pedestal, separatrix). Here, the particular choice is typically determined by the requirement of a simple and coherent description of empirical data and/or ideas about the underlying physics or, sometimes, simply by limitations of the available diagnostics. Historically, the disruptive L-mode DL and the $\mathrm{H}-\mathrm{L}$ transition $\mathrm{H}$-mode DL have been the main focus, but the definitions are general enough to also include recent extensions of the concept such as the Type-I ELM H-mode DL [1].

Investigation of DLs may aim at different targets. One, and probably the most important issue, is the prediction of the actual limit density (critical density) for given machine and discharge parameters. Typically, such information is provided 
in terms of scaling relations for the critical density, which may be purely experimental, model based or a combination of both (semi-empirical models). Alternatively, one may be interested in the details of the limiting process(es). The process that sets the critical density is normally the last step in a chain of processes and, consequently, this part is more demanding and is, therefore, less developed. This is true of the H-mode DL in particular. In this paper, the emphasis is on empirical DL scalings following from JET data. However, models played an important role in the definition of recent JET DL experiments and will also be considered during the interpretation of data.

In an empirical study the underlying database should cover all parameters that may potentially impact on the critical density. Apart from heating power $P_{h}$, toroidal field $B_{t}$, major radius $R$ and safety factor $q_{\psi}$ (or plasma current $I_{p}$ ), which are known to be 'strong' parameters, this would include the plasma aspect ratio $A$, the plasma elongation $\kappa$, upper and lower triangularities $\delta_{u}$ and $\delta_{l}$, respectively, parameters characterizing the impurity content, parameters characterizing the divertor configuration (including double null versus single null), the heating method (NBI versus RF), the fuelling method (gas puffing versus pellet injection), the isotope mix of the fuel gas and possibly other parameters not yet identified as relevant.

In reality, the existing database is far from being complete in this sense. Any attempt to improve the situation has to be guided by ITER needs. In fact, while DLs can be relatively easily avoided in current tokamaks without severe impact on operation, ITER will have to operate at rather specific plasma densities and temperatures (at least for the maximum performance discharges), which are determined by a complex set of technical and physical constraints and any additional limitation may downgrade the performance $[2,3]$. The focus on ITER partly simplifies the situation in that it reduces the number of parameters that have to be considered, but also leads to specific requests for DL-related R\&D. So, for instance, the aspect ratio $A$ and elongation $\kappa$ of ITER will not differ much from those of JET and ASDEX Upgrade and most other current tokamaks. Therefore, no extrapolation is required and the $A$ and $\kappa$ dependences can be ignored. Also, ITER will have a $q_{95}$ of around 3, a range well covered by current tokamak operation.

The main difference from present-day machines will be in size and field and these are the key parameters for extrapolation. To put this aspect into the right perspective, one has to take into account that in standard tokamaks (i.e. tokamaks based on tape-wound pure-tension D-shaped coils with aspect ratios between 3 and 4), one has roughly $B_{t}^{\max }[\mathrm{T}] / R[\mathrm{~m}] \simeq 0.8-1.2$, where $B_{t}^{\max }$ is the maximum field on-axis [4]. Unlike present experimental tokamaks, nextgeneration tokamaks will have to operate routinely at the highest possible $B_{t}$ (at least in the reference scenario), so that the $B_{t}$ and $R$ dependences can be interpreted with the constraint $B_{t} \propto R$. As a consequence, the Greenwald density of a nextgeneration device, which is determined by $B_{t} / R$, would not differ from that in high-field discharges of current tokamaks and the need for extrapolation would not arise. However, in the case of a more complex dependence, both the $R$ and $B_{t}$ dependences become a matter of concern. Improvement of the JET database with respect to $B_{t}$ was one of the main goals of the recent DL related activities at JET. They provided clear evidence that the $B_{t}$ dependence is indeed weaker than the linear dependence predicted by the Greenwald scaling. Thus, the $R$-dependence, which requires data from different machines, becomes an issue. We, therefore, include ASDEX Upgrade data into our analysis.

The role of triangularity is somewhat special. Even the old data included cases with ITER-like triangularities. Unfortunately, the ASDEX Upgrade and JET data were grouped in relatively narrow, disjunct bands, thus constituting a strong covariance between $R$ and $\delta$, which made it virtually impossible to distinguish between the two. Since there is practically no way of varying $R$ in either JET or ASDEX Upgrade, the only way to remedy the situation was to assess the $\delta$ dependence as accurately as possible. Thus, though there is no particular need to extrapolate in $\delta$, investigation of the impact of the plasma shape on the DL is mandatory for reliable size scaling. Extension of triangularity variation in the JET database was, therefore, the second main goal of the recent DL related activities at JET.

Little attention has been paid to the role of impurities, presumably because, apart from simple global parameters like $Z_{\text {eff }}$ and total radiation $P_{\text {rad }}^{\text {tot }}$, it is not obvious how to characterize the impurity population adequately and how to control it. Later, we will replace the heating power by the net input power $P_{\text {net }}=P_{h}-P_{\text {rad }}^{\text {tot }}$, thus taking at least the impact of impurity radiation on the energy balance into account. This choice, to some extent, reflects the view of an edge based DL mechanism underlying this paper.

During the past decade various divertor configurations have been implemented at JET (Mark-I, Mark-II-A, Mark-II-AP (plugged bypasses) [5] and Mark-II-GB (Gas box, with and without septum) [6] $)^{1}$. Data from all configurations are included in our database, and we will make the origin visible in graphical representations. However, since there are no indications of an impact of the divertor geometry on the H-mode DL, we skip the explicit discussion of this aspect and assume no dependence of the H-mode DL on divertor geometry.

The majority of JET data has been obtained in deuterium discharges. A small number of high density hydrogen and deuterium discharges have been analysed in [7] but the number is too small to include them in a statistical analysis. We, therefore, confine ourselves to deuterium discharges so that a possible isotope dependence remains undetermined. Finally, information on other than beam heated discharges is restricted to one recent ICRH gas scan that fits well into the picture of beam heated discharges. Also, this study is confined to gas-fuelled discharges.

Having specified the parameters under consideration the next step is the choice of an appropriate target density. This choice largely depends on the picture of the underlying physics. There is some divergence on this question, but a broad distinction can be made between core and edge oriented pictures. In the first case the central or line-averaged densities might be appropriate [8], while in the second case

\footnotetext{
1 In what follows we ignore some minor modifications of the Mark-II divertor and use, respectively, the notations Mark-II-A and Mark-II-GB for the versions Mark-II-A and AP and the gas box versions with and without septum.
} 
the pedestal [9] or even the separatrix densities [10] might be the right choice. In practice, due to diagnostics limitations, the central line averaged or pedestal densities are the candidates that are available with reasonable accuracy. We generally find that the pedestal density increases monotonically with the gas rate. From a purely empirical point of view this suggests that we conceive the H-mode DL as a limit of the pedestal density that is reached at the $\mathrm{H}-\mathrm{L}$ boundary. In our present database the situation is much simplified in that we always have flat density profiles at the DL so that the central line averaged and pedestal densities coincide. This is an empirical finding that may not be generally true and has to be verified for each particular point in the database.

After these general remarks we discuss ASDEX Upgrade and JET DL signatures in greater detail in sections 2.1 and 2.2 , respectively. The resulting database is described in section 3. Empirical scaling relations are derived in section 4 and compared with model predictions. Extrapolation to ITER is discussed in section 5 .

\section{Density ramp-up signatures}

\subsection{ASDEX Upgrade}

In ASDEX Upgrade the DL is typically approached by ramping up the density within a discharge by feedback controlling the gas rate. The $\mathrm{H}-\mathrm{L}$ transition is regularly reached and coincides with divertor detachment (see figure 1) [11]. There are cases where the discharge disrupts after the $\mathrm{H}-\mathrm{L}$ transition, but in other cases, such as in figure 1 , the density continues to increase in L-mode until the discharge disrupts in a way characteristic of the L-mode DL. It is likely that this behaviour is determined by the relative magnitude of the $\mathrm{H}$-mode and L-mode DLs (under conditions at the $\mathrm{H}-\mathrm{L}$ transition). This topic, which bears some interesting implications on the possibility of non-disruptive shut-down, is, however, beyond the scope of this paper.

Recently, it has become obvious that the core density reorganization times are quite large and can become comparable with the ramp-up time in some discharges [12]. This led to a critical review of the existing database from the point of view to stationarity, which will be discussed in section 3 .

\subsection{JET}

In JET, density ramp-ups are typically realized by performing a sequence of discharges with constant, but successively increasing gas rates. For not too high powers and fields the flat top phase easily exceeds $5 \mathrm{~s}$, sufficient to guarantee the achievement of steady-state conditions. Typical time traces of such a scan can be found in [9].

Figure 2 gives the line-averaged and pedestal densities versus gas rate for a recent medium-field, medium-current gas box divertor scan. It is typical in that $\bar{n}$ saturates (plateau) and eventually shows a mild drop (normally not exceeding 10\%), while $n_{\text {ped }}$ increases monotonically until the H-L boundary is reached. The non-monotonic relation between $\bar{n}$ and $n_{\text {ped }}$ is due to profile flattening as illustrated in figure 3 . From a purely empirical point of view this suggests defining the H-mode DL as a limit of the edge (pedestal) density which is reached at
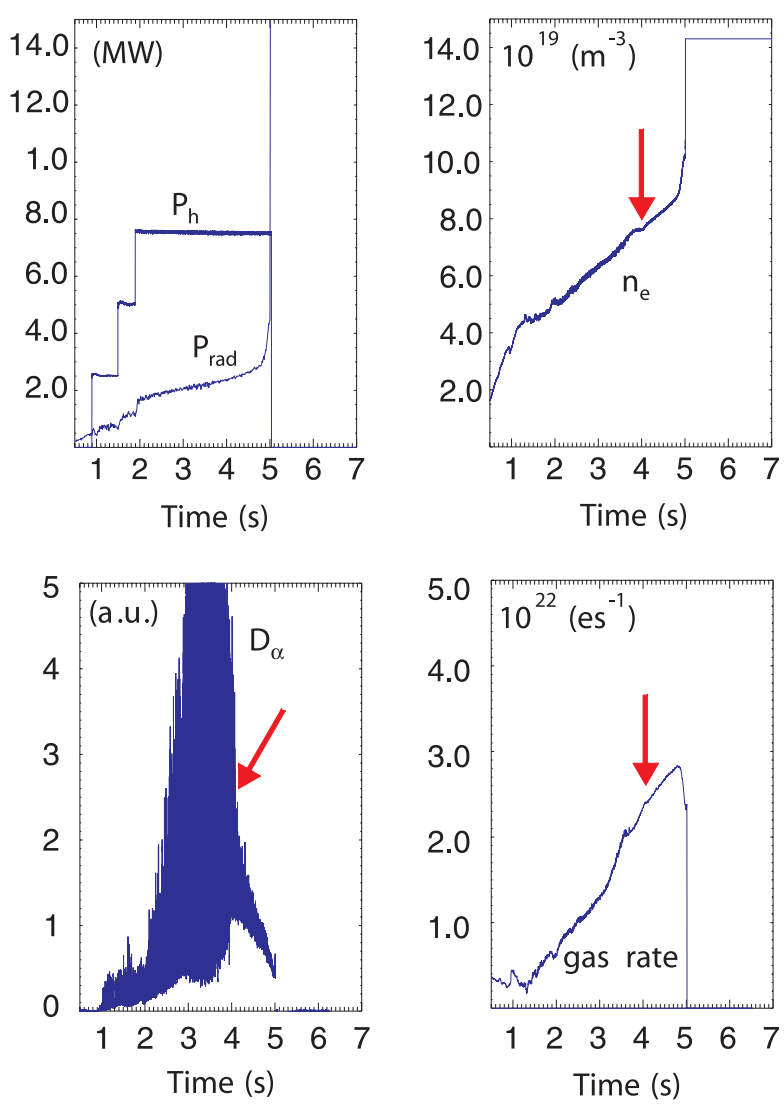

Figure 1. Time traces of, respectively, heating power and total radiative power $P_{h}$ and $P_{\text {rad }}$ (upper left), central line-averaged electron density $n_{e}$ (upper right), $\mathrm{D}_{\alpha}$ signal (lower left) and gas rate (lower right) for ASDEX Upgrade discharge 11158. The arrows indicate the $\mathrm{H}-\mathrm{L}$ transition at $4 \mathrm{~s}$. It is characterized by the drop of the $\mathrm{D}_{\alpha}$ signal and a hesitation of density.

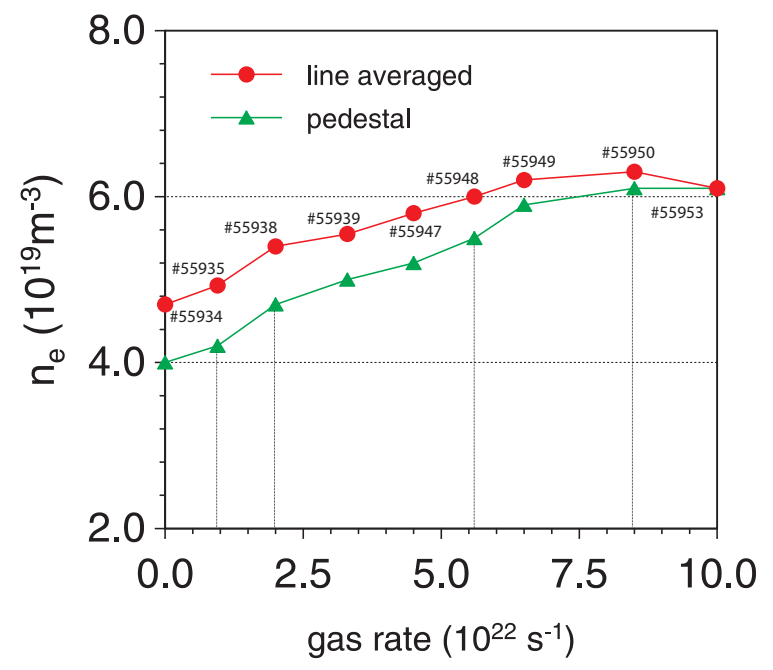

Figure 2. Line-averaged and pedestal densities versus gas rate in a medium-field $\left(B_{t}=2.4 \mathrm{~T}\right)$, medium-power $\left(P_{h}=12 \mathrm{MW}\right)$,

medium- $q_{95}\left(q_{95}=3.7\right)$, medium triangularity $\left(\delta_{u} / \delta_{l}=0.23 / 0.26\right)$ configuration.

the high-density $\mathrm{H}-\mathrm{L}$ transition boundary. Apart from the decrease of the density peaking towards complete flatness, a further generally observed feature of this limit is a drop of the inter-ELM ion saturation current $I_{\text {sat }}$ by typically one order 


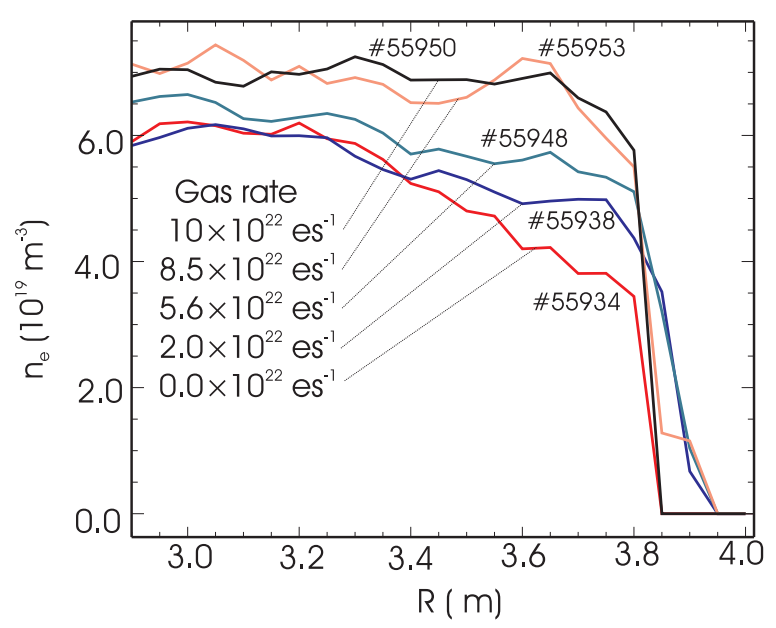

Figure 3. Electron density profiles (Lidar) for various discharges of the gas scan of figure 2 .

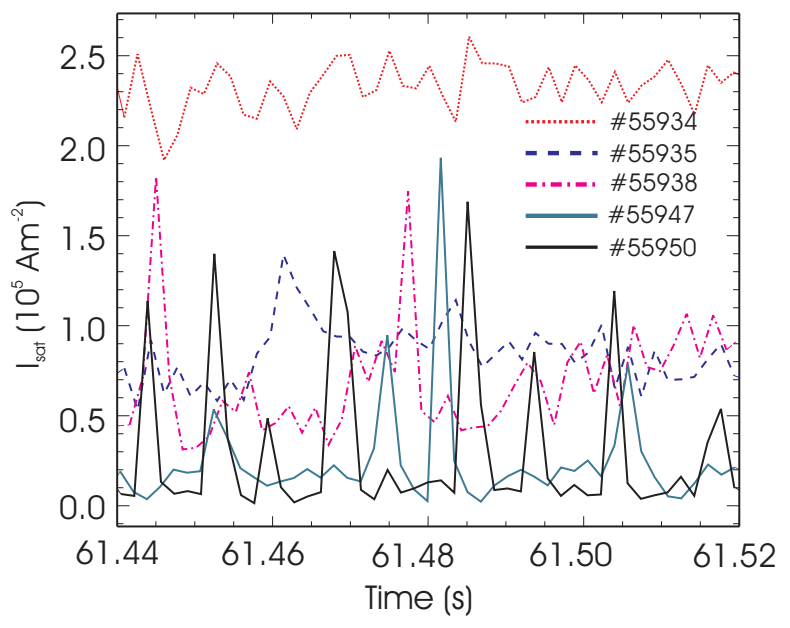

Figure 4. Ion saturation current time traces of an inboard, near strike point Langmuir probe for various discharges of the gas scan of figure 2. The inter-ELM ion saturation current drops by more than an order of magnitude. (For the two low gas rate cases, which have low ELM frequencies, no ELMs occur in the chosen time window.)

of magnitude (see figure 4), this being indicative of divertor detachment between ELMs [13].

By adopting the pedestal density as target density one obtains a picture for the H-mode DL in JET which is in line with findings from ASDEX Upgrade [11]. This then allowed us to combine data from the two machines. The only difference, namely the non-monotonic behaviour of $\bar{n}$, is naturally attributed to differences in core peaking which are likely to be governed by core physics.

Though the picture given is perfectly adequate for the data we use, the situation is actually more complicated. For high current, high triangularity cases a drop of $n_{\text {ped }}$ is observed with increasing gas rate, when the Type-I to Type-III (I-III) boundary is crossed. Unfortunately, for currents above about 2.5 MA the gas rates required to reach the H-L boundary exceed the limit set by the maximum allowable beam duct pressure. It, therefore, remains unclear, whether $n_{\text {ped }}$ would recover on the way to the $\mathrm{H}-\mathrm{L}$ boundary to values exceeding the I-III density. The physics at the two boundaries is certainly different. The situation is, therefore, most adequately
Table 1. Main parameters of recent JET H-mode DL gas scans ${ }^{\mathrm{a}}$.

\begin{tabular}{llllrll}
\hline & $\begin{array}{l}B_{t} \\
{[\mathrm{~T}]}\end{array}$ & $q_{95}$ & $\begin{array}{l}I_{p} \\
{[\mathrm{MA}]}\end{array}$ & $\begin{array}{l}P_{h} \\
{[\mathrm{MW}]}\end{array}$ & $\begin{array}{l}\delta_{\mathrm{av}} \\
\end{array}$ & $\begin{array}{l}n_{\mathrm{DL}} \\
{\left[10^{19} \mathrm{~m}^{-3}\right]}\end{array}$ \\
\hline 50570 & 1.9 & 2.9 & 1.9 & 7.7 & 0.19 & 6.3 \\
52294 & 1.5 & 2.8 & 1.5 & 5.1 & 0.20 & 5.7 \\
52300 & 2.6 & 5.0 & 1.5 & 8.9 & 0.19 & 4.7 \\
52304 & 2.6 & 5.0 & 1.5 & 8.3 & 0.20 & 5.0 \\
55950 & 2.4 & 3.8 & 2.0 & 11.0 & 0.24 & 6.3 \\
55929 & 1.2 & 3.2 & 1.2 & 5.7 & 0.30 & 4.3 \\
55974 & 2.4 & 3.7 & 2.0 & 9.5 & 0.31 & 6.4 \\
57006 & 1.3 & 3.4 & 1.2 & 5.7 & 0.32 & 4.6 \\
58410 & 2.7 & 3.3 & 2.5 & 9.5 & 0.30 & 6.9 \\
58418 & 2.4 & 3.8 & 2.0 & 15.0 & 0.31 & 6.3 \\
58820 & 1.2 & 3.4 & 1.2 & 7.0 & 0.41 & 4.4 \\
59648 & 2.4 & 4.0 & 2.0 & 11.0 & 0.24 & 5.7 \\
\hline
\end{tabular}

${ }^{\text {a }} B_{t}, q_{95}, I_{p}, P_{h}, \delta$ and $n_{\mathrm{DL}}$ are, respectively, the toroidal field on-axis, the safety factor at the $95 \%$ flux surface, the plasma current, the heating power, the mean triangularity and the central line-averaged density at the limit. Discharge numbers given are those within a scan that are at or closest to the $\mathrm{H}-\mathrm{L}$ transition.

interpreted in terms of two competing limiting mechanisms with (at least slightly) different scaling, but occurring at comparable critical densities. In the light of the limitations discussed, and since there is little information on the scaling of the I-III density, it is currently impossible to predict whether and under what conditions the I-III density may exceed the $\mathrm{H}-\mathrm{L}$ density. Investigation of the I-III boundary is beyond the scope of this paper.

\section{Discussion of the database}

\subsection{Physics aspects}

Between 2000 and 2003 a series of dedicated H-mode density limit discharges were performed in the context of the issues discussed so far. A total number of 12 complete scans have been performed, the main parameters of which are summarized in table 1 . The new data extend the existing database mainly towards low $B_{t}$ and high $\delta$, but some high $q_{95}$ points were also added. In fact, most earlier JET gas scans were focused on $q_{95}$ values between 3 and 4 . Though this would be sufficient as regards extrapolation to ITER, a better assessment of the $q$-dependence would greatly help in discriminating between existing scalings which differ, apart from the $B_{t}$ and $R$ dependences, mainly in their $q_{95}$ dependence.

Unfortunately, most earlier JET high-density gas scans fail to reach the high-density H-L boundary ('incomplete scans'). Since, however, the drop in the final phase is small and the density profile at the limit is flat, we have with acceptable accuracy $\bar{n}_{\text {plateau }} \simeq \bar{n}_{\mathrm{DL}} \simeq n_{\text {ped,DL }} \equiv n_{\mathrm{DL}}$, where the subscript DL indicates values at the DL proper and the meanings of the other notations are obvious. This offered the possibility of including older, incomplete scans that reach the plateau in our database by identifying $n_{\mathrm{DL}}$ with $\bar{n}_{\text {plateau }}$. The selection criteria applied were: at least two or three subsequent discharges with nearly constant $\bar{n}$ and, to rule out fortuitous results, gas rates above and density peaking below certain thresholds. This is, of course, a purely empirical approach, which requires a representative set of complete scans (in comparable parameter ranges) for its validation. Such scans existed for traditional 
medium triangularity scans $(\delta \approx 0.2)$ in sufficient number. On the other hand, though high triangularity scans had been performed prior to the recent campaign as a consequence of the increased interest in high triangularity configurations, they were all incomplete. With this problem in mind, all scans of table 1 , and the high triangularity ones in particular, were designed to actually reach the $\mathrm{H}-\mathrm{L}$ boundary. (This limits the accessible range of plasma currents to values below about 2.5 MA for the reasons discussed in the previous section.) With the information gathered in this way, all old data were revisited with a view to the proximity to the $\mathrm{H}-\mathrm{L}$ boundary. This included Mark-I and II data of $[9,11]$ plus some additional scans that were performed between the compilation of [11] and the installation of the gas box divertor. This exercise led to the removal of a major fraction of old points. So, for instance, all data of [9], except one, were discarded. In total our database now includes 23 JET points (2 Mark-I, 5 Mark-II and 16 gas box cases, including the ones of table 1).

Having established a representative set of complete scans in all relevant parameter ranges, we find that the use of scans of varying degree of completeness introduces additional random errors, comparable to the intrinsic ones. Thus, it is not surprising that the scalings to be derived in section 4 from the purified database, where comparable, do not differ significantly from earlier findings $[9,11]$. However, apart from the aspect of methodological purity, strict confinement to complete scans reduces the scatter of our data by about a factor of two resulting in smaller confidence intervals in the scalings and more reliable estimates for ITER.

Our main interest is the high-density $\mathrm{H}-\mathrm{L}$ transition boundary in contrast to the (conventional) medium density $\mathrm{H}-\mathrm{L}$ threshold. (For a nice graphic visualization of these various regimes see, for instance, [11].) In order to avoid the medium density or transition regimes in a ramp-up, the power has to be well above the medium density $\mathrm{H}-\mathrm{L}$ power threshold under conditions of the high-density $\mathrm{H}-\mathrm{L}$ transition. All JET cases meet this requirement.

As pointed out, we include ASDEX Upgrade data to establish the size dependence of the DL. Basically, these are the points in [11]. However, some discharges with a rapid variation of both power and density were omitted due to concerns about stationarity. In ASDEX Upgrade the medium density $\mathrm{H}-\mathrm{L}$ power threshold and the high-density $\mathrm{H}-\mathrm{L}$ transition boundary are conveniently characterized by the parameter combination $P_{h} / I_{p} B_{t}$ introduced in [11]. Cases with $P_{h} / I_{p} B_{t}<3$ [MW, MA, T], indicative of the transition regime, were also removed. Thus, the number of ASDEX Upgrade points was reduced to 19 .

According to the discussion of section 1, one should seek a scaling in terms of the major radius $R$, shaping (upper and lower triangularities $\delta_{u}$ and $\delta_{l}$, respectively), toroidal field $B_{t}$, safety factor $q_{95}$, heating power $P_{h}$ and radiated power or other impurity related parameters. As mentioned earlier, we take potentially different impurity levels into account by replacing the heating power by the net input power $P_{\text {net }}=$ $P_{h}-P_{\text {rad }}^{\text {tot }}$, where $P_{\text {rad }}^{\text {tot }}$ is the total radiated power. Since the power dependence will be found to vanish virtually and since radiative fractions have typical values in the $40 \%$ range, this is a detail that has little impact. Finally, $P_{\text {net }}$ is replaced by the mean power flux across the separatrix $q_{\perp}\left(q_{\perp}=P_{\text {net }} / O_{p}\right.$, where $O_{p}$ is the plasma surface) to simplify the discussion of section 4.2. Variations of $\delta_{u}$ and/or $\delta_{l}$ suffer from numerous device-specific constraints. In the light of these limitations it seems appropriate to characterize triangularity, as a first step, by a single parameter such as the mean triangularity $\delta_{\mathrm{av}}=\left(\delta_{u}+\delta_{l}\right) / 2$. In summary, we then seek a scaling of $n_{\text {crit }}$ in terms of $R, B_{t}, q_{95}, q_{\perp}$ and $\delta_{\text {av }}$.

\subsection{Statistics aspects}

Anticipating a critical density scaling of the power law type, we will apply the usual log-linear regression approach. Collinearity of the regression variables may seriously hamper the reliable evaluation of the regression coefficients as well as the interval estimate for ITER. We discuss these aspects along the lines that have been developed in connection with the assessment of the global energy confinement database for ITER [14-16]. A key element of this approach is the principal components method. Interpreting each data point as a vector (five components in our case), the principal components are the components in a new basis (derived variables), defined by the requirement of a diagonal covariance matrix. Conventionally, the new basis vectors are labelled such that the eigenvalues of the covariance matrix (which are equal to the variances in the derived variables) decrease with increasing label.

Collinearities are unavoidable and some occurring in the problem under discussion have been mentioned in previous sections. However, they do not affect the reliability of a regression provided that the variation in the data is sufficiently large with respect to the measurement errors. Quantitatively, this means that

$$
\frac{\sigma_{P i}}{4 \sigma_{\mathrm{diag}, i}}>1
$$

where $\sigma_{P i}$ and $\sigma_{\text {diag }, i}$ are, respectively, the standard deviation of the $i$ th principal component and $\sigma_{\text {diag }, i}$ the standard deviation of the non-systematic measurement errors in the same direction $[14,16]$. We assume that the standard deviations of the nonsystematic measurement errors in $R, B_{t}, q_{95}, q_{\perp}$ and $\delta_{\mathrm{av}}$ are $1 \%, 1 \%, 2 \%, 5 \%$ and $2 \%$, respectively. With this input the condition equation (1) is well fulfilled for all principal components (see table 2).

\section{Scaling considerations}

\subsection{Empirical scaling}

From the log-linear regression we now obtain the empirical scaling

$$
n_{\mathrm{DL}, \mathrm{fit}}=48.2 \frac{q_{\perp}^{0.049 \pm 0.041} B_{t}^{0.57 \pm 0.08} \delta_{\mathrm{av}}^{0.11 \pm 0.11}}{q_{95}^{0.86 \pm 0.08} R^{1.07 \pm 0.20}}
$$

$\left[10^{19} \mathrm{~m}^{-3}, \mathrm{MW} \mathrm{m}{ }^{-2}, \mathrm{~T}, \mathrm{~m}\right]$, where the exponents are given with their $95 \%$ confidence intervals. The standard deviation is found to be $\sigma=0.0525$.

Equation (2) is complemented by information from figure 5. Figure 5 provides the range of $q_{\perp}, B_{t}, q_{95}$ and $\delta_{\mathrm{av}}$ variations covered by the database. Most gas box points result from the recent dedicated scans. They considerably extended the parameters towards high $q_{95}$, low $B_{t}$ and to high $\delta_{\mathrm{av}}$ in particular. Figure 5 also provides some check of the power 
Table 2. Summary of principal components analysis ${ }^{\mathrm{a}}$.

\begin{tabular}{|c|c|c|c|c|c|c|c|c|}
\hline \multicolumn{9}{|c|}{ Full parameter set } \\
\hline & $\ln (R)$ & $\ln \left(B_{t}\right)$ & $\ln \left(q_{95}\right)$ & $\ln \left(q_{\perp}\right)$ & $\ln \left(\delta_{\mathrm{av}}\right)$ & $\sigma_{P}$ & $\frac{\sigma_{P}}{4 \sigma_{\text {diag }}}$ & $\frac{\Delta_{\mathrm{ITER}}}{\sigma_{P}}$ \\
\hline P1 & 0.2672 & -0.04873 & -0.07194 & -0.9075 & 0.3123 & 0.97 & 5.3 & 0.17 \\
\hline P2 & -0.3692 & -0.7602 & 0.05571 & -0.2362 & -0.4762 & 0.31 & 4.5 & 4.6 \\
\hline P3 & 0.1312 & -0.5292 & -0.5795 & 0.2951 & 0.5290 & 0.28 & 3.1 & 0.55 \\
\hline P4 & -0.04513 & 0.3092 & -0.8048 & -0.1335 & -0.4865 & 0.19 & 2.4 & 0.22 \\
\hline P5 & 0.8792 & -0.2096 & 0.09038 & 0.1257 & -0.3988 & 0.072 & 1.3 & 6.9 \\
\hline \multicolumn{9}{|c|}{ Restricted parameter set } \\
\hline & $\ln \left(q_{95} R\right)$ & $\ln \left(B_{t}\right)$ & $\ln \left(q_{\perp}\right)$ & $\ln \left(\delta_{\mathrm{av}}\right)$ & & $\sigma_{P}$ & $\frac{\sigma_{P}}{4 \sigma_{\text {diag }}}$ & $\frac{\Delta_{\mathrm{ITER}}}{\sigma_{P}}$ \\
\hline P1 & 0.2111 & -0.04778 & -0.9252 & 0.3117 & & 0.96 & 12.0 & 0.058 \\
\hline P2 & -0.5242 & -0.7829 & -0.1754 & -0.2856 & & 0.33 & 4.9 & 4.3 \\
\hline P3 & -0.03358 & -0.3665 & 0.3070 & 0.8777 & & 0.2 & 3.2 & 1.0 \\
\hline P4 & 0.8244 & -0.5005 & 0.1379 & -0.2257 & & 0.18 & 2.1 & 0.82 \\
\hline
\end{tabular}

a The $5 \times 5$ and $4 \times 4$ matrices formed by the first five and four columns, respectively, are the transformation matrices from the original to the derived coordinates. In the last three columns $\sigma_{P i}$ are the standard deviations of the $i$ th component, $\sigma_{\text {diag }, i}$ are the standard deviations of the non-systematic measurement errors in the $i$ th direction (see text) and $\Delta_{\mathrm{ITER}, i}$ is the $i$ th component of the distance of the ITER point from the centre of gravity of the database (to be used in section 5) [14].
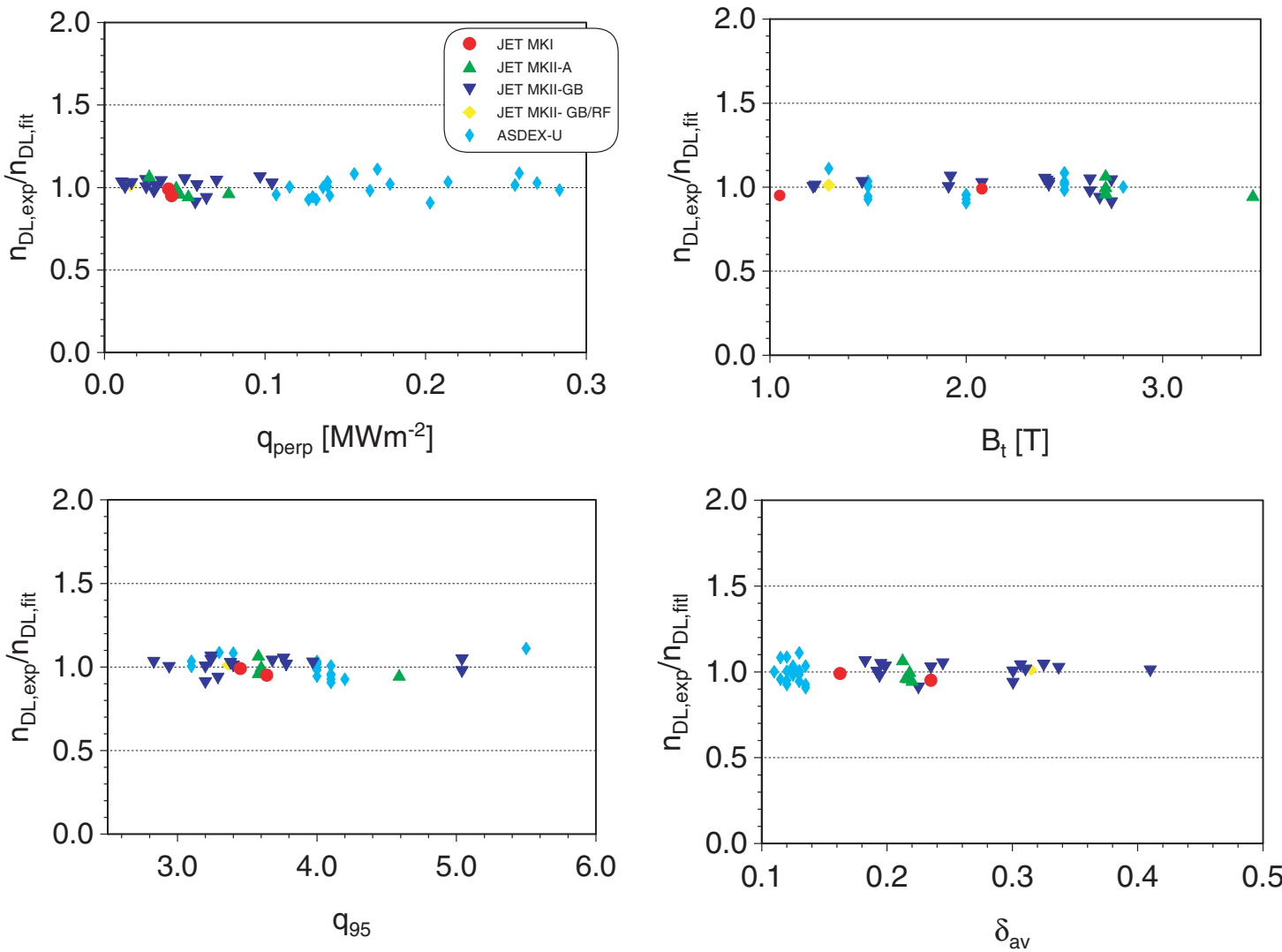

Figure 5. Experimental critical densities $n_{\text {DL,exp }}$ from JET and ASDEX-Upgrade over $n_{\text {DL,fit }}$ calculated from equation (1) versus mean power flux across the separatrix $q_{\perp}$, toroidal field $B_{t}$, safety factor at the $95 \%$ flux surface $q_{95}$ and mean triangularity $\delta_{\text {av }}$. (In all four figures some points are so close that they become indistinguishable on the current scale and resolution, possibly giving the misleading impression that the number of points differs from the figures quoted in the text. However, through the particular choice of the axis interval one ASDEX Upgrade point at $q_{95}=11.5$ is suppressed in the $q_{95}$ trace.)

law ansatz underlying the log-linear regression. In fact, any non-linear effect (e.g. regime transitions) would be exhibited as deviations from the 'horizontal band' structure of the cloud of points in a plot like figure 5. Provided that the scales are suitably chosen, such deviations can be reliably detected by visual inspection. In this sense figure 5 confirms that the power law ansatz underlying equation (2) is indeed justified for all variables. One can also verify that there are no indications of scaling differences between the JET and ASDEX Upgrade subsets. 
In existing scalings, to be discussed in the subsequent section, the $q_{95}$ and $R$-dependences enter through the combination $q_{95} R$. It is interesting to repeat the regression analysis for this restricted format. By analogy with equation (2) we then get

$$
n_{\text {DL,fit }}=39.5 \frac{q_{\perp}^{0.079 \pm 0.032} B_{t}^{0.53 \pm 0.07} \delta_{\mathrm{av}}^{0.014 \pm 0.068}}{\left(q_{95} R\right)^{0.87 \pm 0.08}}
$$

The $\delta$-dependence virtually vanishes. Having reduced the number of variables, the standard deviation must be higher, but the increase to $\sigma=0.0552$ is modest. The increase in $\sigma$ is statistically significant, if [16]

$$
\frac{\Delta \sigma}{\sigma}>\frac{1.92}{N}
$$

where $N$ is the number of data points. In our case $\Delta \sigma / \sigma \simeq$ $(0.0552-0.0525) / 0.052 \simeq 0.051$. For the right-hand side we get $1.92 / 42 \simeq 0.046$. Thus the gain by treating $R$ and $q_{95}$ as independent variables is only marginally significant. ASDEX Upgrade data in a wider triangularity range would help to clarify this issue.

\subsection{Comparison with existing scalings}

As discussed in section 2, the attainment of the DL coincides with complete inter-ELM divertor detachment. Thus, the model for the (pedestal) density $n_{\mathrm{DL}, \mathrm{BLS}}$ at complete detachment proposed by Borrass et al [9] should describe our data. It results in the scaling (equation (7) of [9])

$$
n_{\mathrm{DL}, \mathrm{BLS}}=40.6 \frac{q_{\perp}^{0.094} B_{t}^{0.53}}{\left(q_{95} R\right)^{0.88}}
$$

$\left[10^{19} \mathrm{~m}^{-3}, \mathrm{MW} \mathrm{m}{ }^{-2}, \mathrm{~T}, \mathrm{~m}\right]^{2}$. The agreement between equations (3) and (5) is remarkable.

As a second example we consider the empirical Greenwald scaling, which is widely used as a reference scaling [17]:

$$
n_{\mathrm{DL}, \mathrm{GW}}=10 \frac{I_{p}}{\pi a^{2}} \equiv 15.9 g \frac{B_{t}}{q_{95} R}
$$

$\left[10^{19} \mathrm{~m}^{-3}, \mathrm{MA}, \mathrm{T}, \mathrm{m}\right]$, where $I_{p}$ is the plasma current and $a$ the plasma minor radius. The factor $g=q_{95} / q_{c}\left(q_{c}=\right.$ $\left(2 \pi / \mu_{0}\right)\left(a^{2} B_{t} / R I_{p}\right)$ the cylindrical $\left.q\right)$ is determined by the plasma shape.

The quality of the various scalings considered is compared in figure 6. As one would suspect from the previous discussion, the Borrass, Lingertat, Schneider (BLS) scaling and the empirical scaling are hardly distinguishable. The Greenwald densities are in the right absolute range, but the overall fit is rather poor.

\section{Extrapolation to ITER}

Table 3 summarizes the critical densities and Greenwald fractions as predicted by equations (2)-(6) for the present ITER-FEAT parameters $\left(R=6.2 \mathrm{~m}, a=2.0 \mathrm{~m}, B_{t}=5.3 \mathrm{~T}\right.$, $I_{p}=15.0 \mathrm{MA}, q_{95}=3.2, \delta_{\mathrm{av}}=0.33, q_{\perp}=0.044$ (estimated

2 With the improved database the empirical coefficient in equation (5) decreases from its value of 41.4 in [9] to 40.6 .
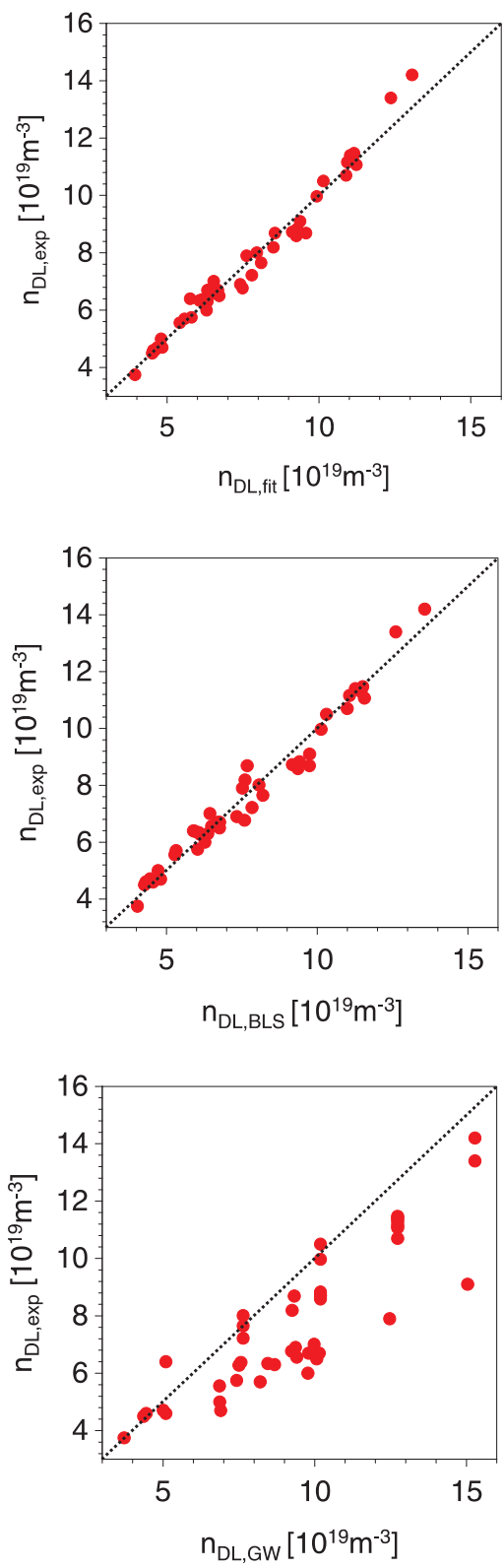

Figure 6. Experimental critical density $n_{\mathrm{DL}, \exp }$ versus $n_{\mathrm{DL} \text {, fit }}$ according to equation (2) (top), $n_{\mathrm{DL}, \mathrm{BLS}}$ (middle) and Greenwald density $n_{\mathrm{DL}, \mathrm{GW}}$ (bottom). (Same data as in figure 5).

from $P_{\alpha}=80 \mathrm{MW}, P_{h}=40 \mathrm{MW}, P_{\mathrm{rad}}^{\text {tot }}=90 \mathrm{MW}(400 \mathrm{MW}$ inductive scenario) and a plasma surface of $\left.680 \mathrm{~m}^{2}\right)$ ) [18]. Also given are the power law prediction errors for the full and restricted scalings. The $95 \%$ confidence intervals are estimated with the expression $[16,15]$

$$
\frac{\delta_{n_{\text {crit }}}}{n_{\text {crit }}}=\frac{2 \sigma}{\sqrt{N}}\left(1+\sum_{i}\left(\frac{\Delta_{\mathrm{ITER}, i}}{\sigma_{P i}}\right)^{2}\right)^{1 / 2}
$$

where $\delta_{n_{\text {crit }}}$ is the half-width of the confidence interval, $\sigma$ the standard deviation of the regression, $\Delta_{\text {ITER }, i}$ the $i$ th component (in the derived system) of the distance from ITER to the centre of gravity of the database, $\sigma_{P i}$ the standard deviation of the $i$ th principal component (see table 2) and $N$ the number of data points. 
Table 3. Critical ITER-FEAT densities according to the various scalings considered.

\begin{tabular}{llcll}
\hline Scaling & Equation & $n_{\text {crit }}\left[10^{19} \mathrm{~m}^{-3}\right]$ & {$\left[n_{\min }, n_{\max }\right]^{\mathrm{a}}$} & $f_{G}{ }^{\mathrm{b}}$ \\
\hline Empirical/full & Equation (2) & 4.9 & {$[4.3,5.6]$} & 0.41 \\
Empirical/restricted & Equation (3) & 5.5 & {$[4.7,6.3]$} & 0.46 \\
BLS & Equation (5) & 5.4 & - & 0.45 \\
Greenwald & Equation (6) & 12.0 & - & 1 \\
\hline
\end{tabular}

a $95 \%$ confidence interval. ${ }^{\mathrm{b}}$ Greenwald fraction.

The critical densities and Greenwald fractions, resulting from equations (2) to (5), are well below the ITER reference values of $10.1 \times 10^{19} \mathrm{~m}^{-3}$ and 0.85 , respectively [18]. Also, the reference value is outside the confidence intervals predicted for both empirical scalings.

At present, it is difficult to decide precisely what this would mean for the operation of ITER. Following the ITER plasma performance analysis, Greenwald fractions of at least 0.7 are required to achieve $Q=10$, the primary objective of ITER, for the reference confinement scaling [18].

Core fuelling, which may offer the possibility of controlling the line-averaged density irrespective of limits existing for pedestal and/or separatrix densities, is considered as an option to alleviate the situation. So far, the focus has been on two different variants of this concept, namely (i) central pellet injection and (ii) deep fuelling (i.e. pellet injection just beyond the ELM-affected region). The first scheme aims at core density peaking, while the basic idea of the second one is to control the pedestal density by generating a controllable particle flux into the pedestal. Particle transport in the core and pedestal are potentially different so that both schemes have to be validated separately. Core density peaking associated with central pellet fuelling has been successfully demonstrated in current tokamaks including JET [19]. The ITER fuelling concept instead relies on a combination of gas puffing and deep fuelling by pellet injection from the high field side [18]. This concept has been extensively studied in a series of papers [20-22]. These rather involved and selfconsistent simulations of SOL and core inherently rely on a number of transport models, currently partly semi-empirical or purely heuristic. A discussion of this concept is beyond the scope of this paper. However, it is probably not unfair to say that this is a conceivable solution, awaiting its empirical validation.

\section{Summary and conclusions}

Recent JET DL studies were reported. The main aim of these experiments was, besides the clarification of some underlying concepts, an assessment of the $B_{t}$ and $R$-dependences. The extrapolation to ITER is largest in $B_{t}$ and $R$ and a knowledge of their dependence is mandatory for any prediction.

As a first step, the density ramp-up signatures were rediscussed with the main conclusion that the majority of earlier gas scans were incomplete in that they did not reach the $\mathrm{H}-\mathrm{L}$ boundary. All recent scans were designed to be complete. With a representative number of complete scans available the role of profile effects for the divergent behaviour of line-averaged and pedestal densities could be clarified. This led to the definition of the H-mode DL as a limit of pedestal density which is reached at the $\mathrm{H}-\mathrm{L}$ boundary. This definition is in line with edge based models for the H-mode DL. Divertor detachment is a central element of these models. Direct empirical evidence for detachment at the limit was provided for JET complete scans.

In order to assess the size dependence, data from different machines have to be considered and, therefore, ASDEX Upgrade data were included in the analysis. With the above DL definition complete coherence between JET and ASDEX Upgrade DL signatures was found and this allowed us to perform scaling studies on the combined JET and ASDEX Upgrade database. However, strong covariance between size and triangularity was observed in the existing data, which made it virtually impossible to distinguish between the two dependences. A major fraction of the experiments was, therefore, dedicated to triangularity scans to determine the shape dependence as accurately as possible.

From the extended database an empirical scaling was derived applying the usual log-linear regression. The condition of the database was checked and found to be satisfactory. Most significant with regard to ITER are the weaker than linear $B_{t}$ dependence and a divergence between the $R$ and $B_{t}$ dependences. The triangularity dependence, if any, is weak.

A nested database, where the variables $R$ and $q_{95}$ are replaced by $q_{95} R$, suggested by model derived scalings (restricted scaling), results in a scaling which is practically identical to the detachment based BLS scaling. On the database all scalings provide fits which are virtually indistinguishable. This complements the direct observation of complete divertor detachment at the $\mathrm{H}-\mathrm{L}$ boundary and provides additional support for the role of detachment as a triggering mechanism for the high density $\mathrm{H}-\mathrm{L}$ transition. The Greenwald scaling predicts critical densities in the right absolute range, but the overall fit is rather poor.

These findings also help to put results from previous studies of the JET H-mode DL in the right perspective. In [11] an empirical scaling was derived where the parameter choice was triggered by the BLS model. Not surprising in the light of what was said above, it is not too different from what is derived in this paper. However, apart from the improved quality of the database, this paper goes far beyond earlier studies in that it provides for the first time a purely empirical scaling in all relevant parameters including, in particular, $R$ and $B_{t}$.

While the conditions of the database are satisfactory from a statistical point of view, some caveats have to be made relating to non-statistical aspects. So, for instance, as a consequence of the restriction to two devices, any systematic difference between the two machines would manifest itself through an impact on the $R$-dependence. This could be in the form of unrecognized physics effects, systematic measurement errors, profile effects, etc. The latter could play a role for the relatively old ASDEX Upgrade data. Repetition of some measurements, 
preferably including higher triangularities, in the present much better diagnosed machine would help clarify this point. (Note, however, that any residual peaking, when taken into account, would shift the results in a direction, unfavourable for ITER.)

Among the parameters not well covered so far, the isotope dependence and the impact of various heating methods are probably the most important ones. Improvements in this area would come from a more systematic investigation of hydrogen discharges and discharges with RF or combined RF and beam heating.

For all these reasons the inclusion of data from additional tokamaks would be desirable. Such a broader campaign would require considerable effort, probably comparable to the assessment of the energy confinement.

For the present ITER parameters the predictions are substantially below the Greenwald value $(\approx 50 \%)$ and the ITER nominal density $(\approx 40 \%)$. The lowest density allowing operation at $Q=10$ would be outside the confidence interval estimate for the empirical scalings [18]. The situation would be much alleviated if core and SOL density could be controlled independently by combined gas puffing and deep fuelling as proposed for ITER [18]. Currently, this concept has to largely rely on models. Tests of the underlying computational tools on data from present machines (such as the ones used in this paper) in the limit of pure gas puffing are easily conceivable. An effort in this direction could be an important step towards a validation of this concept.

The non-monotonic evolution of the pedestal density with the gas rate in the vicinity of the I-III transition observed at JET at high current and high triangularity may also offer some additional room for optimism. Whether the pedestal density at the I-III boundary may actually exceed the pedestal density at the $\mathrm{H}-\mathrm{L}$ boundary in JET is an open question and difficult to verify directly due to the inaccessibility of the $\mathrm{H}-\mathrm{L}$ boundary in the parameter range of interest. Probably a careful assessment of the scaling of the I-III pedestal density, which exists only in rudimentary form, is required to clarify this point. Such an exercise would also be mandatory to estimate the relevance for
ITER. In the light of the somewhat discouraging results of this study, this aspect should be pursued with priority.

\section{Acknowledgment}

The authors gratefully acknowledge advice from O. Kardaun on statistics issues.

\section{References}

[1] Chankin A.V. and Saibene G. 1999 Plasma Phys. Control. Fusion 41913

[2] ITER Physics Basis Editors 1999 ITER Physics Expert Group Chairs and Co-Chairs, ITER Joint Central Team and Physics Integration Unit Nucl. Fusion 392137

[3] Kardaun O.J.W.F. 2002 Nucl. Fusion 42841

[4] Borrass K. 1987 Nucl. Eng. Des./Fusion 4247

[5] Horton L.D. et al 1999 Nucl. Fusion 391

[6] Altmann H. et al 1997 Proc. 19th Symp. on Fusion Technology 1996 (SOFT), (Lisbon, 1996) vol 1 (Amsterdam: North-Holland) p 483

[7] Saibene G. et al 1999 Nucl. Fusion 391133

[8] Greenwald M. 2002 Plasma Phys. Control. Fusion 44 R27

[9] Borrass K., Lingertat J. and Schneider R. 1998 Contrib. Plasma Phys. 38130

[10] Borrass K., Schneider R. and Farengo R. 1997 Nucl. Fusion 37523

[11] Mertens V. et al 2000 Nucl. Fusion 401839

[12] Stober J. et al 2002 Plasma Phys. Control. Fusion 44 A159

[13] Borrass K., Coster D., Reiter D. and Schneider R. 1997 J. Nucl. Mater 241-243 250

[14] Christiansen J.P. et al 1992 Nucl. Fusion 32291

[15] Cordey J.G. et al 1997 Plasma Phys. Control. Fusion 39 B115-27

[16] Kardaun O.J.W.F. 1999 Plasma Phys. Control. Fusion 41429

[17] Greenwald M. et al 1988 Nucl. Fusion 282199

[18] ITER Technical Basis 2002 ITER EDA Documentation Series No 24 (Vienna: IAEA)

[19] Lang P.T. et al 2002 Plasma Phys. Control. Fusion 441919

[20] Pacher G.W. et al 2003 Nucl. Fusion 43188

[21] Kukushkin A.S. et al 2003 Nucl. Fusion 43716

[22] Kukushkin A.S. et al 2002 Plasma Phys. Control. Fusion 44931 